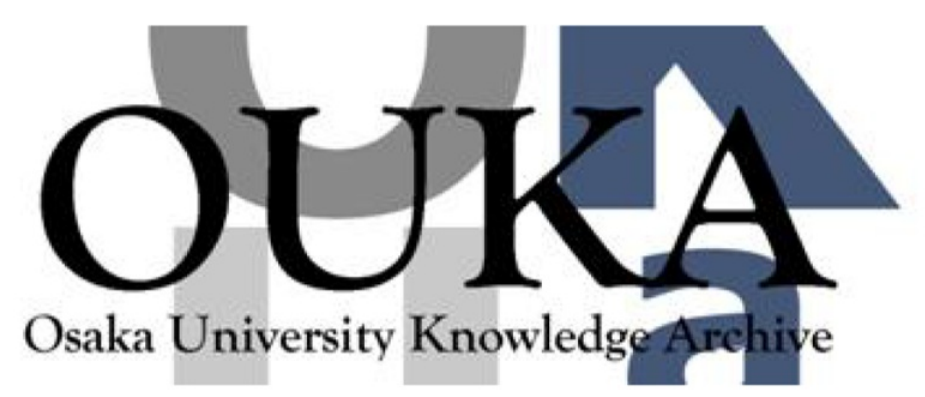

\begin{tabular}{|c|l|}
\hline Title & $\begin{array}{l}\text { Evaluation of Interaction Parameters in Dilute } \\
\text { Liquid Ternary Alloys by a Solution Model Based } \\
\text { on the Free Volume Theory }\end{array}$ \\
\hline Author(s) & $\begin{array}{l}\text { Tanaka, Toshihiro; Gokcen, Nev A.; Spencer, } \\
\text { Philip J. et al. }\end{array}$ \\
\hline Citation & Zeitschrift für Metallkunde. 84(2) p. 100-p. 105 \\
\hline Issue Date & 1993 \\
\hline oaire:version & VoR \\
\hline URL & https://hdl. handle. net/11094/26510 \\
\hline rights & oCarl Hanser Verlag, München \\
\hline Note & \\
\hline
\end{tabular}

Osaka University Knowledge Archive : OUKA

https://ir. Library. osaka-u. ac. jp/

Osaka University 
Toshihiro Tanaka*, Nev A. Gokcen**, Philip J. Spencer***, Zen-Ichiro Morita* and Takamichi Iida*

(* Department of Materials Science and Processing, Faculty of Engineering, Osaka University, 2-1 Yamadaoka, Suita, Osaka, 565, Japan, ** Albany Research Center, U. S. Bureau of Mines, 1450 Queen Avenue SW, Albany, Oregon 97321-2198 USA, and *** Lehrstuhl für Theoretische Hüttenkunde und Metallurgie der Kernbrennstoffe, RWTH Aachen, Kopernikusstraße 16, 5100 Aachen, Germany)

\section{Evaluation of Interaction Parameters in Dilute Liquid Ternary Alloys by a Solution Model Based on the Free Volume Theory}

A solution model based on the free volume theory has been applied to derive a Gibbs energy interaction parameter $\varepsilon_{\mathrm{B}}^{\mathrm{C}}$. The relation between an enthalpy interaction parameter $\eta_{\mathrm{B}}^{\mathrm{C}}$ and an entropy interaction parameter $\sigma_{\mathrm{B}}^{\mathrm{C}}$ has been obtained. These parameters were evaluated from some physical properties coupled with Miedema's semi-empirical method.

\section{Introduction}

In previous publications [ 1 to 5], a new solution model for liquid binary alloys based on the free volume theory has been presented. This model allows a successful description of the relation between partial enthalpy and partial excess entropy of mixing in infinitely dilute solutions of liquid binary alloys. In addition, it predicts the values of partial excess Gibbs energy of solutions, which are in reasonable agreement with experimental values.

In the present work, this solution model has been applied to ternary alloys to evaluate Gibbs energy interaction parameters in infinite dilution. Moreover, the relation between enthalpy and entropy interaction parameters is discussed.

\section{Derivation of Thermodynamic Equations}

The solution model is based on the free volume theory advanced by Shimoji and Niwa [6]. Assuming that an atom vibrates harmonically in its cell surrounded by the nearest-neighbor atoms, the partition functions for pure liquid $\mathrm{A}, \mathrm{B}$ and $\mathrm{C}$ is given by the following equation:

$$
\begin{aligned}
Q_{X X} & =V_{\mathrm{F}, X}^{N_{X}} \cdot \exp \left(-E_{X} / k T\right) \\
& =\left\{-\pi L_{X X}^{2} k T / U_{X X}\right\}^{3 N_{X} / 2} \cdot \exp \left(-N_{X} U_{X X} / 2 k T\right) \\
& (X X=\mathrm{AA}, \mathrm{BB} \text { or } \mathrm{CC} ; X=\mathrm{A}, \mathrm{B} \text { or } \mathrm{C})
\end{aligned}
$$

where

$U_{X X}=z \cdot u_{X X}$

$V_{\mathrm{F}, X}$ is the free volume of $X$ atom in pure liquid $(X=\mathrm{A}, \mathrm{B}$ or C);
$U_{X X}$ is the depth of potential energy of an atom $X(X=\mathrm{A}, \mathrm{B}$ or $\mathrm{C}$ ) in its cell in pure liquid $X$, as shown in Fig. 1;

$u_{\mathrm{XX}}$ is the energy of A-A, B-B or C-C pairs;

$z \quad$ is the coordination number;

$L_{X X}$ is the distance which interatomic potential extends in a cell of pure liquid $\mathrm{A}, \mathrm{B}$ or $\mathrm{C}$, as shown in Fig. 1;

$N_{X}$ is the number of $X$ atom $(X=\mathrm{A}, \mathrm{B}$ or $\mathrm{C})$;

$k$ is the Boltzmann constant and

$T$ is the temperature in $\mathrm{K}$.

The partition function for an A-B-C ternary alloy is

$$
\begin{aligned}
& Q=g \cdot\left\{-\pi L_{\mathrm{A}}^{2} k T / U_{\mathrm{A}}\right\}^{3 N_{\mathrm{A}} / 2} \cdot\left\{-\pi L_{\mathrm{B}}^{2} k T / U_{\mathrm{B}}\right\}^{3 N_{\mathrm{B}} / 2} \\
& \cdot\left\{-\pi L_{\mathrm{C}}^{2} k T / U_{\mathrm{C}}\right\}^{3 N_{\mathrm{C}} / 2} \\
& \cdot \exp \left\{-\left(N_{\mathrm{A}} U_{\mathrm{A}}+N_{\mathrm{B}} U_{\mathrm{B}}+N_{\mathrm{C}} U_{\mathrm{C}}\right) / 2 k T\right\}
\end{aligned}
$$

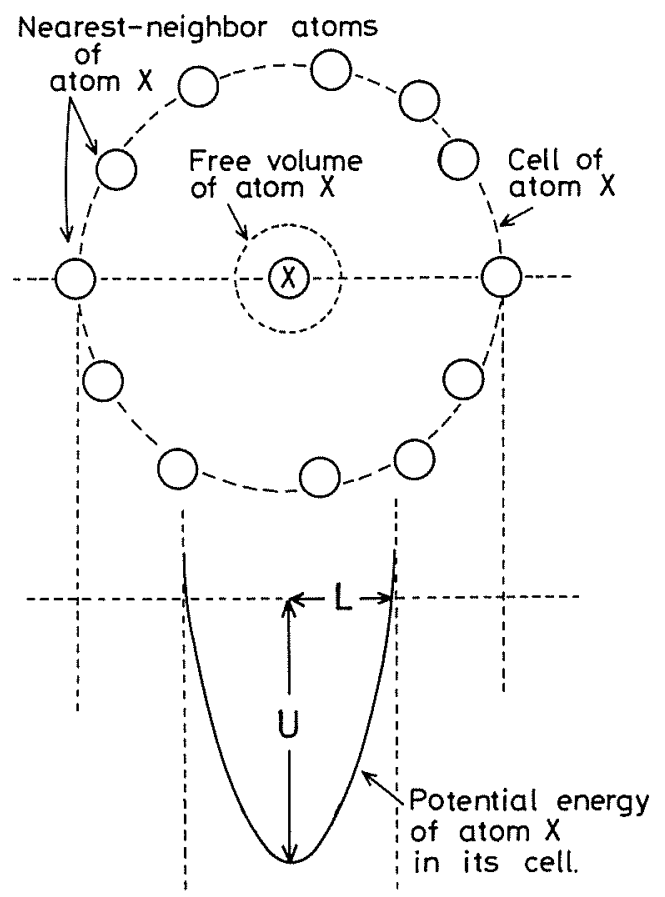

Fig. 1. Schematic diagram of potential energy in a cell of atom $X$. 
where

$g$

is a degeneracy factor;

$U_{X}(X=\mathrm{A}, \mathrm{B}$ or $\mathrm{C})$ the depth of potential energy of an atom $X$ $(X=\mathrm{A}, \mathrm{B}$ or $\mathrm{C})$ in its cell in liquid $\mathrm{A}-\mathrm{B}-\mathrm{C}$ ternary alloy and

$L_{X}(X=\mathrm{A}, \mathrm{B}$ or $\mathrm{C})$ the distance which interatomic potential extends in a cell of an atom $X(X=\mathrm{A}, \mathrm{B}$ or $C)$ in liquid A-B-C ternary alloy.

The energy $E=\left(N_{\mathrm{A}} U_{\mathrm{A}}+N_{\mathrm{B}} U_{\mathrm{B}}+N_{\mathrm{C}} U_{\mathrm{C}}\right) / 2$ of an A-B-C ternary alloy in Eq. (3) is assumed to be expressed in terms of the pair interaction energies $\mathrm{A}-\mathrm{A}, \mathrm{B}-\mathrm{B}, \mathrm{C}-\mathrm{C}, \mathrm{A}-\mathrm{B}, \mathrm{A}-\mathrm{C}$ and $\mathrm{B}-\mathrm{C}$, namely $u_{\mathrm{AA}}, u_{\mathrm{BB}}, u_{\mathrm{CC}}, u_{\mathrm{AB}}, u_{\mathrm{AC}}$ and $u_{\mathrm{BC}}$ as follows:

$$
\begin{aligned}
E= & N_{\mathrm{A}} U_{\mathrm{AA}} / 2+N_{\mathrm{B}} U_{\mathrm{BB}} / 2+N_{\mathrm{C}} U_{\mathrm{CC}} / 2 \\
& +N_{\mathrm{AB}} \Omega_{\mathrm{AB}} / z+N_{\mathrm{BC}} \Omega_{\mathrm{BC}} / z+N_{\mathrm{AC}} \Omega_{\mathrm{AC}} / z
\end{aligned}
$$

where $U_{\mathrm{AA}}=z \cdot u_{\mathrm{AA}}, U_{\mathrm{BB}}=z \cdot u_{\mathrm{BB}}$ and $U_{\mathrm{CC}}=z \cdot u_{\mathrm{CC}}$

$N_{\mathrm{AB}}, N_{\mathrm{AC}}$ and $N_{\mathrm{BC}}$ in Eq. (4) are the numbers of A-B, A-C and $\mathrm{B}-\mathrm{C}$ pairs, respectively. $\Omega_{\mathrm{AB}}, \Omega_{\mathrm{BC}}$ and $\Omega_{\mathrm{AB}}$ are defined as follows:

$$
\begin{aligned}
& \Omega_{\mathrm{AB}}=z \cdot\left\{u_{\mathrm{AB}}-1 / 2\left(u_{\mathrm{AA}}+u_{\mathrm{BB}}\right)\right\} \\
& \Omega_{\mathrm{BC}}=z \cdot\left\{u_{\mathrm{BC}}-1 / 2\left(u_{\mathrm{BB}}+u_{\mathrm{CC}}\right)\right\} \\
& \Omega_{\mathrm{AC}}=z \cdot\left\{u_{\mathrm{AC}}-1 / 2\left(u_{\mathrm{AA}}+u_{\mathrm{CC}}\right)\right\}
\end{aligned}
$$

For condensed systems and at ordinary pressure levels, the Gibbs energy $G$ may be equivalent to the Helmholtz energy $F$ because their difference, the term $P V$, is usually negligible. Thus, for all practical purpose

$$
G_{X X} \sim F_{X X}=-k T \ln Q_{X X} \quad(X X=\mathrm{AA}, \mathrm{BB} \text { or } \mathrm{CC})
$$

$$
G \sim F=-k T \ln Q
$$

From the above equations, the change in Gibbs energy of mixing, $\Delta G_{\mathrm{MIX}}$ is

$$
\begin{aligned}
\Delta G_{\mathrm{MIX}}= & G-\left(G_{\mathrm{AA}}+G_{\mathrm{BB}}+G_{\mathrm{CC}}\right) \\
= & \left(N_{\mathrm{AB}} \Omega_{\mathrm{AB}}+N_{\mathrm{BC}} \Omega_{\mathrm{BC}}+N_{\mathrm{AC}} \Omega_{\mathrm{AC}}\right) / z-k T \cdot \ln g \\
& -k T \cdot 3 / 2\left[N_{\mathrm{A}} \cdot \ln \left(-\pi L_{\mathrm{A}}^{2} k T / U_{\mathrm{A}}\right)+\right. \\
& N_{\mathrm{B}} \cdot \ln \left(-\pi L_{\mathrm{B}}^{2} k T / U_{\mathrm{B}}\right)+N_{\mathrm{C}} \cdot \ln \left(-\pi L_{\mathrm{C}}^{2} k T / U_{\mathrm{C}}\right) \\
& -\left\{N_{\mathrm{A}} \cdot \ln \left(-\pi L_{\mathrm{AA}}^{2} k T / U_{\mathrm{AA}}\right)+\right. \\
& N_{\mathrm{B}} \cdot \ln \left(-\pi L_{\mathrm{BB}}^{2} k T / U_{\mathrm{BB}}\right)+ \\
& \left.\left.N_{\mathrm{C}} \cdot \ln \left(-\pi L_{\mathrm{CC}}^{2} k T / U_{\mathrm{CC}}\right)\right\}\right]
\end{aligned}
$$

In the following, the enthalpy and entropy terms in the A-B-C ternary alloy will be derived separately.

\subsection{The Enthalpy Term}

From Eq. (8),

$\Delta H_{\mathrm{MIX}}^{\prime}=\left(N_{\mathrm{AB}} \Omega_{\mathrm{AB}}+N_{\mathrm{BC}} \Omega_{\mathrm{BC}}+N_{\mathrm{AC}} \Omega_{\mathrm{AC}}\right) / z$

With regard to the configuration of atoms in alloys, short range order is taken into account on the basis of the first approximation to the regular solution model proposed by one of the authors [7]. According to this model, $N_{\mathrm{AB}}, N_{\mathrm{BC}}$ and $N_{\mathrm{AC}}$ are given by the following equations for dilute solutions:
$N_{\mathrm{AB}}=z \cdot N_{0} \cdot x_{\mathrm{A}} x_{\mathrm{B}}\left(1-x_{\mathrm{A}} x_{\mathrm{B}} \Omega_{\mathrm{AB}} / k T\right)$

$N_{\mathrm{BC}}=z \cdot N_{0} \cdot x_{\mathrm{B}} x_{\mathrm{C}}\left(1-x_{\mathrm{B}} x_{\mathrm{C}} \Omega_{\mathrm{BC}} / k T\right)$

$N_{\mathrm{AC}}=z \cdot N_{0} \cdot x_{\mathrm{A}} x_{\mathrm{C}}\left(1-x_{\mathrm{A}} x_{\mathrm{C}} \Omega_{\mathrm{AC}} / k T\right)$

where

$N_{0} \quad$ is Avogadro's number,

$x_{\mathrm{A}}, x_{\mathrm{B}}$ and $x_{\mathrm{C}}$ are the mole fractions of $\mathrm{A}, \mathrm{B}$ and $\mathrm{C}$, respectively.

Consequently, the enthalpy of mixing per atom $\Delta H_{\mathrm{MTX}}=\Delta H_{\mathrm{MIX}}^{\prime} / N_{0}$ can be given by the following equation:

$$
\begin{aligned}
\Delta H_{M I X} & =\Omega_{\mathrm{AB}} \cdot x_{\mathrm{A}} \cdot x_{\mathrm{B}}\left(1-x_{\mathrm{A}} x_{\mathrm{B}} \Omega_{\mathrm{AB}} / k T\right) \\
& +\Omega_{\mathrm{BC}} \cdot x_{\mathrm{B}} \cdot x_{\mathrm{C}}\left(1-x_{\mathrm{B}} x_{\mathrm{C}} \Omega_{\mathrm{BC}} / k T\right) \\
& +\Omega_{\mathrm{AC}} \cdot x_{\mathrm{A}} \cdot x_{\mathrm{C}}\left(1-x_{\mathrm{A}} x_{\mathrm{C}} \Omega_{\mathrm{AC}} / k T\right)
\end{aligned}
$$

\subsection{Entropy Term}

The entropy arising from the atomic configuration in the alloys, $\Delta S_{\mathrm{CONF}}=k \cdot \ln g$, consists of the following two terms:

$$
\Delta S_{\mathrm{CONF}}=k \cdot \ln g=\Delta S_{\mathrm{CONF}}^{\mathrm{Ideal}}+\Delta S_{\mathrm{CONF}}^{\mathrm{Ex}}
$$

For the excess configurational entropy in the alloys, the following equation based on the first approximation to the regular solution model [7] was also adopted.

$$
\begin{aligned}
\Delta S_{\mathrm{CONF}}^{\mathrm{Ex}} & =-x_{\mathrm{A}}^{2} x_{\mathrm{B}}^{2} \Omega_{\mathrm{AB}}^{2} / 2 k T^{2}-x_{\mathrm{B}}^{2} x_{\mathrm{C}}^{2} \Omega_{\mathrm{BC}}^{2} / 2 k T^{2} \\
& -x_{\mathrm{A}}^{2} x_{\mathrm{C}}^{2} \Omega_{\mathrm{AC}}^{2} / 2 k T^{2}
\end{aligned}
$$

The excess entropy arising from nonconfigurational contributions, for example, from the vibration of atoms in their cell, $\Delta S_{\mathrm{VIB}}^{\mathrm{Ex}}$ can be derived from Eq. (8) as follows:

$$
\begin{aligned}
\Delta S_{\mathrm{VIB}}^{\mathrm{Ex}^{\prime}=} & 3 / 2 \cdot k N_{0}\left\{2 x_{\mathrm{A}} \cdot \ln \left(L_{\mathrm{A}} / L_{\mathrm{AA}}\right)+\right. \\
& 2 x_{\mathrm{B}} \cdot \ln \left(L_{\mathrm{B}} / L_{\mathrm{BB}}\right)+2 x_{\mathrm{C}} \cdot \ln \left(L_{\mathrm{C}} / L_{\mathrm{CC}}\right)+ \\
& x_{\mathrm{A}} \cdot \ln \left(U_{\mathrm{AA}} / U_{\mathrm{A}}\right)+x_{\mathrm{B}} \cdot \ln \left(U_{\mathrm{BB}} / U_{\mathrm{B}}\right)+ \\
& \left.x_{\mathrm{C}} \cdot \ln \left(U_{\mathrm{CC}} / U_{\mathrm{C}}\right)\right\}
\end{aligned}
$$

In Eq. (16), it is necessary to have information on $L_{\mathrm{A}}, L_{\mathrm{B}}, L_{\mathrm{C}}, U_{\mathrm{A}}, U_{\mathrm{B}}$ and $U_{\mathrm{C}}$ in the A-B-C ternary alloy. In this work, the simplest relations were adopted from Shimoji and Niwa [6], i.e.,

$$
\begin{aligned}
& U_{\mathrm{A}}=x_{\mathrm{A}} U_{\mathrm{AA}}+x_{\mathrm{B}} U_{\mathrm{AB}}+x_{\mathrm{C}} U_{\mathrm{AC}} \\
& U_{\mathrm{B}}=x_{\mathrm{A}} U_{\mathrm{AB}}+x_{\mathrm{B}} U_{\mathrm{BB}}+x_{\mathrm{C}} U_{\mathrm{BC}} \\
& U_{\mathrm{C}}=x_{\mathrm{A}} U_{\mathrm{AC}}+x_{\mathrm{B}} U_{\mathrm{BC}}+x_{\mathrm{C}} U_{\mathrm{CC}}
\end{aligned}
$$

where

$U_{\mathrm{AB}}=z \cdot u_{\mathrm{AB}} \quad U_{\mathrm{BC}}=z \cdot u_{\mathrm{BC}} \quad U_{\mathrm{AC}}=z \cdot u_{\mathrm{AC}}$

$L_{\mathrm{A}}=1 / 2\left\{L_{\mathrm{AA}}+\left(x_{\mathrm{A}} L_{\mathrm{AA}}+x_{\mathrm{B}} L_{\mathrm{BB}}+x_{\mathrm{C}} L_{\mathrm{CC}}\right)\right\}$

$L_{\mathrm{B}}=1 / 2\left\{L_{\mathrm{BB}}+\left(x_{\mathrm{A}} L_{\mathrm{AA}}+x_{\mathrm{B}} L_{\mathrm{BB}}+x_{\mathrm{C}} L_{\mathrm{CC}}\right)\right\}$

$L_{\mathrm{C}}=1 / 2\left\{L_{\mathrm{CC}}+\left(x_{\mathrm{A}} L_{\mathrm{AA}}+x_{\mathrm{B}} L_{\mathrm{BB}}+x_{\mathrm{C}} L_{\mathrm{CC}}\right)\right\}$

Finally, the excess entropy for vibrational contributions of atoms in their cell per atom, $\Delta S_{\mathrm{VIB}}^{\mathrm{Ex}}=\Delta S_{\mathrm{VIB}}^{\mathrm{Ex}} / N_{0}$ is given by 


$$
\begin{aligned}
\Delta S_{\mathrm{VIB}}^{\mathrm{Ex}} & =3 / 2 k x_{\mathrm{A}} x_{\mathrm{B}}\left[\left(L_{\mathrm{AA}}-L_{\mathrm{BB}}\right)^{2} /\left(L_{\mathrm{AA}} \cdot L_{\mathrm{BB}}\right)\right. \\
& +\left\{4 U_{\mathrm{AA}} U_{\mathrm{BB}}-2 \Omega_{\mathrm{AB}}\left(U_{\mathrm{AA}}+U_{\mathrm{BB}}\right)\right. \\
& \left.\left.-\left(U_{\mathrm{AA}}+U_{\mathrm{BB}}\right)^{2}\right\} /\left(2 U_{\mathrm{AA}} U_{\mathrm{BB}}\right)\right] \\
& +3 / 2 k x_{\mathrm{B}} x_{\mathrm{C}}\left[\left(L_{\mathrm{BB}}-L_{\mathrm{CC}}\right)^{2} /\left(L_{\mathrm{BB}} \cdot L_{\mathrm{CC}}\right)\right. \\
& +\left\{4 U_{\mathrm{BB}} U_{\mathrm{CC}}-2 \Omega_{\mathrm{BC}}\left(U_{\mathrm{BB}}+U_{\mathrm{CC}}\right)\right. \\
& \left.\left.-\left(U_{\mathrm{BB}}+U_{\mathrm{CC}}\right)^{2}\right\} /\left(2 U_{\mathrm{BB}} U_{\mathrm{CC}}\right)\right] \\
& +3 / 2 k x_{\mathrm{A}} x_{\mathrm{C}}\left[\left(L_{\mathrm{AA}}-L_{\mathrm{CC}}\right)^{2} /\left(L_{\mathrm{AA}} \cdot L_{\mathrm{CC}}\right)\right. \\
& +\left\{4 U_{\mathrm{AA}} U_{\mathrm{CC}}-2 \Omega_{\mathrm{AC}}\left(U_{\mathrm{AA}}+U_{\mathrm{CC}}\right)\right. \\
& \left.\left.-\left(U_{\mathrm{AA}}+U_{\mathrm{CC}}\right)^{2}\right\} /\left(2 U_{\mathrm{AA}} U_{\mathrm{CC}}\right)\right]
\end{aligned}
$$

where the following relations were used:

$$
\begin{aligned}
& U_{\mathrm{AB}}=\Omega_{\mathrm{AB}}+\left(U_{\mathrm{AA}}+U_{\mathrm{BB}}\right) / 2 \\
& U_{\mathrm{BC}}=\Omega_{\mathrm{BC}}+\left(U_{\mathrm{BB}}+U_{\mathrm{CC}}\right) / 2 \\
& U_{\mathrm{AC}}=\Omega_{\mathrm{AC}}+\left(U_{\mathrm{AA}}+U_{\mathrm{CC}}\right) / 2
\end{aligned}
$$

The first order Gibbs energy interaction parameter $\varepsilon_{\mathrm{B}}^{\mathrm{C}}$, proposed by Wagner [8], may be written as

$$
\begin{aligned}
\varepsilon_{\mathrm{B}}^{\mathrm{C}} & =\left\{\left(\partial^{2} \Delta G^{\mathrm{Ex}} / \partial x_{\mathrm{C}} \partial x_{\mathrm{B}}\right)_{x_{\mathrm{B}} \rightarrow 0, x_{\mathrm{C}} \rightarrow 0}\right\} / k T \\
& =\left(\eta_{\mathrm{B}}^{\mathrm{C}}-T \sigma_{\mathrm{B}}^{\mathrm{C}}\right) / \hat{k} T
\end{aligned}
$$

where $\eta_{\mathrm{B}}^{\mathrm{C}}$ and $\sigma_{\mathrm{B}}^{\mathrm{C}}$ are the enthalpy and entropy interaction parameters, respectively, and according to Lupis and Elliott [9] are defined as follows:

$$
\begin{aligned}
\eta_{\mathrm{B}}^{\mathrm{C}} & =\left(\partial^{2} \Delta H_{\mathrm{MIX}} / \partial x_{\mathrm{C}} \partial x_{\mathrm{B}}\right)_{x_{\mathrm{B}} \rightarrow 0, x_{\mathrm{C}} \rightarrow 0} \\
\sigma_{\mathrm{B}}^{\mathrm{C}} & =\left(\partial^{2} \Delta S^{\mathrm{Ex}} / \partial x_{\mathrm{C}} \partial x_{\mathrm{B}}\right)_{x_{\mathrm{B}} \rightarrow 0, x_{\mathrm{C}} \rightarrow 0} \\
& =\left\{\partial^{2}\left(\Delta S_{\mathrm{CONF}}^{\mathrm{Ex}}+\Delta S_{\mathrm{VIB}}^{\mathrm{Ex}}\right) / \partial x_{\mathrm{C}} \partial x_{\mathrm{B}}\right\}_{x_{\mathrm{B}} \rightarrow 0, x_{\mathrm{C}} \rightarrow 0}
\end{aligned}
$$

From Eqs. (13), (15), (20), (23) and (24), $\eta_{\mathrm{B}}^{\mathrm{C}}$ and $\sigma_{\mathrm{B}}^{\mathrm{C}}$ can be written as

$$
\begin{aligned}
\eta_{\mathrm{B}}^{\mathrm{C}}= & \Omega_{\mathrm{BC}}-\Omega_{\mathrm{AB}}-\Omega_{\mathrm{AC}} \\
\sigma_{\mathrm{B}}^{\mathrm{C}}= & 3 / 2 k\left[\left(L_{\mathrm{BB}}-L_{\mathrm{CC}}\right)^{2} /\left(L_{\mathrm{BB}} L_{\mathrm{CC}}\right)+\left\{4 U_{\mathrm{BB}} U_{\mathrm{CC}}\right.\right. \\
& \left.-2 \Omega_{\mathrm{BC}}\left(U_{\mathrm{BB}}+U_{\mathrm{CC}}\right)-\left(U_{\mathrm{BB}}+U_{\mathrm{CC}}\right)^{2}\right\} /\left(2 U_{\mathrm{BB}} U_{\mathrm{CC}}\right) \\
& -\left(L_{\mathrm{AA}}-L_{\mathrm{BB}}\right)^{2} /\left(L_{\mathrm{AA}} L_{\mathrm{BB}}\right)-\left\{4 U_{\mathrm{AA}} U_{\mathrm{BB}}\right. \\
& \left.-2 \Omega_{\mathrm{AB}}\left(U_{\mathrm{AA}}+U_{\mathrm{BB}}\right)-\left(U_{\mathrm{AA}}+U_{\mathrm{BB}}\right)^{2}\right\} /\left(2 U_{\mathrm{AA}} U_{\mathrm{BB}}\right) \\
& -\left(L_{\mathrm{AA}}-L_{\mathrm{CC}}\right)^{2} /\left(L_{\mathrm{AA}} L_{\mathrm{CC}}\right)-\left\{4 U_{\mathrm{AA}} U_{\mathrm{CC}}\right. \\
& \left.\left.-2 \Omega_{\mathrm{AC}}\left(U_{\mathrm{AA}}+U_{\mathrm{CC}}\right)-\left(U_{\mathrm{AA}}+U_{\mathrm{CC}}\right)^{2}\right\} /\left(2 U_{\mathrm{AA}} U_{\mathrm{CC}}\right)\right]
\end{aligned}
$$

From Eqs. (22), (25) and (26), the following relation can be obtained.

$$
\varepsilon_{\mathrm{B}}^{\mathrm{C}}=\varepsilon_{\mathrm{C}}^{\mathrm{B}}
$$

Further, $\varepsilon_{\mathrm{B}}^{\mathrm{B}}$ can be expressed as follows:

$\varepsilon_{\mathrm{B}}^{\mathrm{B}}=\left(\eta_{\mathrm{B}}^{\mathrm{B}}-T \sigma_{\mathrm{B}}^{\mathrm{B}}\right) / k T$

$\eta_{\mathrm{B}}^{\mathrm{B}}=-2 \Omega_{\mathrm{AB}}$

$$
\begin{aligned}
\sigma_{\mathrm{B}}^{\mathrm{B}}= & -3 k\left[\left(L_{\mathrm{AA}}-L_{\mathrm{BB}}\right)^{2} / L_{\mathrm{AA}} L_{\mathrm{BB}}\right. \\
& +\left\{4 U_{\mathrm{AA}} U_{\mathrm{BB}}-2 \Omega_{\mathrm{AB}}\left(U_{\mathrm{AA}}+U_{\mathrm{BB}}\right)\right. \\
& \left.\left.-\left(U_{\mathrm{AA}}+U_{\mathrm{BB}}\right)^{2}\right\} /\left(2 U_{\mathrm{AA}} U_{\mathrm{BB}}\right)\right]
\end{aligned}
$$

According to the solution model, as applied to binary alloys in our previous work [2 to 5], the relation between $\varepsilon_{\mathrm{B}}^{\mathrm{B}}$ and the activity coefficient at infinite dilution $\gamma_{\mathrm{B}}^{0}$ can be expressed by

$\varepsilon_{\mathrm{B}}^{\mathrm{B}}=-2 \ln \gamma_{\mathrm{B}}^{0}$

$L_{X X}(X X=\mathrm{AA}, \mathrm{BB}$ or CC) in Eq. (26) is assumed to be half of the nearest- neighbor distance and is obtained from the following equation [1 to 5]:

$L_{X X}=1 / 2 \cdot\left(2^{1 / 2} V_{X} / N_{0}\right)^{1 / 3}$

where $V_{X}$ is a molar volume of pure $X(\mathrm{X}=\mathrm{A}, \mathrm{B}$ or $\mathrm{C})$.

$\Omega_{\mathrm{BC}}, \Omega_{\mathrm{AB}}$ and $\Omega_{\mathrm{AC}}$ can be assumed to be equal to the partial enthalpy of mixing in infinite dilution $\Delta \bar{H}_{\mathrm{C}(\mathrm{B})}$ of solute $\mathrm{C}$ in solvent $\mathrm{B}, \Delta \bar{H}_{\mathrm{B}(\mathrm{A})}$ of solute $\mathrm{B}$ and $\Delta \bar{H}_{\mathrm{C}(\mathrm{A})}$ of solute $\mathrm{C}$ in solvent $\mathrm{A}$, respectively [2 to 5].

$U_{\mathrm{AA}}, U_{\mathrm{BB}}$ and $U_{\mathrm{CC}}$ in Eq. (26) are calculated from the following equation [4 to 5] when the units of $\Omega_{\mathrm{BC}}, \Omega_{\mathrm{AB}}$ and $\Omega_{\mathrm{AC}}$ are taken as $\mathrm{J} \cdot \mathrm{mol}^{-1}$, and $k$ in Eqs. (1) to (30) is taken as gas constant $R / \mathrm{J} \cdot \mathrm{K}^{-1} \cdot \mathrm{mol}^{-1}$.

$U_{X X}=-685.3 \cdot\left(\beta_{X}\right)^{2} \cdot T_{\mathrm{m}, \mathrm{X}} \quad / \mathrm{J} \cdot \mathrm{mol}^{-1}$

where $T_{\mathrm{m}, X}$ is the melting point of element $X$ and $\beta_{X}$ the coefficient to transfer the solid state frequency to that in the liquid at the melting point [10 to 12 ].

The values of $\beta_{X}$ for various elements were obtained from the experimental data on surface tension of pure liquid metals [10]. When the values of $\beta_{X}$ are not available, it can be set approximately $[10,12]$ to 0.5 .

The derivation of the above Eq. (33) and the physical meaning of $\beta_{X}$ were described in detail in our previous work [ 1 to 5 , 10 to 12$]$.

Consequently, $\eta_{\mathrm{B}}^{\mathrm{C}}$ and $\sigma_{\mathrm{B}}^{\mathrm{C}}$ can be obtained from Eqs. (25) to (33), together with the partial enthalpy of mixing in infinite dilution and some fundamental physical properties, i.e., $T_{\mathrm{m}, X}, V_{X}$ and $\beta_{X}$ of the components in the pure state.

\section{Calculation of the Gibbs Energy Interaction Param- eter, the Enthalpy Interaction Parameter and the Entropy Interaction Parameter}

As shown in Eqs. (4), (13) and (25), $\Delta H_{\mathrm{MIX}}$ in ternary alloys according to the present model consists of the summation of the interaction energies between each pair of elements. Extra terms describing the interactions between the three elements in ternary systems, however, are not considered. If each binary in the ternary system shows large interaction energies, i. e. large absolute values of $\Delta H_{\mathrm{MIX}}$, it seems inappropriate to apply the present model for the calculation of $\varepsilon_{\mathrm{B}}^{\mathrm{C}}$ values. The calculation of $\varepsilon_{\mathrm{B}}^{\mathrm{C}}, \eta_{\mathrm{B}}^{\mathrm{C}}$ and $\sigma_{\mathrm{B}}^{\mathrm{C}}$, therefore, was carried out for non-ferrous ternary alloys formed from $\mathrm{Bi}, \mathrm{Cd}, \mathrm{In}, \mathrm{Pb}, \mathrm{Sb}, \mathrm{Sn}$ and $\mathrm{Zn}$ and also in $\mathrm{Fe}$ $\mathrm{Mn}$ base and $\mathrm{Fe}-\mathrm{Cr}$ base alloys. The components in these systems have similar physical properties and the interaction energies of each pair do not seem to be too great.

The values of $T_{\mathrm{m}, X}, V_{X}$ and $\beta_{X}$ for various elements are shown in Table $1[10,13]$. To evaluate $\Omega_{\mathrm{BC}}, \Omega_{\mathrm{AB}}$ and $\Omega_{\mathrm{AC}}$ in Eqs. (25) and (26), the values of partial enthalpy of mixing $\Delta \bar{H}_{\mathrm{C}(\mathrm{B})}$ in solvent $\mathrm{B}$, and $\Delta \bar{H}_{\mathrm{B}(\mathrm{A})}$ and $\Delta \bar{H}_{\mathrm{C}(\mathrm{A})}$ in solvent $\mathrm{A}$ were calculated using Miedema's semi-empirical method [13], as shown in Table 2. 
Table 1. Physical properties of some elements used in the calculation of $\sigma_{\mathrm{B}}^{\mathrm{C}} \cdot T_{\mathrm{m}, X}: \operatorname{Ref}$. [10], $V_{X}:$ Ref. [13] and $\beta_{X}:$ Ref. [10].

\begin{tabular}{|c|c|c|c|}
\hline Elements & $T_{\mathrm{m}, X} / \mathrm{K}$ & $V_{X} / \mathrm{cm}^{3} \mathrm{~mol}^{-1}$ & $\beta_{X}$ \\
\hline $\mathrm{Bi}$ & 544 & 19.32 & 0.54 \\
$\mathrm{Cd}$ & 594 & 13.00 & 0.56 \\
$\mathrm{Ce}$ & 1068 & 21.62 & 0.50 \\
$\mathrm{Co}$ & 1765 & 6.69 & 0.48 \\
$\mathrm{Cr}$ & 2178 & 7.23 & 0.50 \\
$\mathrm{Cu}$ & 1356 & 7.12 & 0.46 \\
$\mathrm{Fe}$ & 1808 & 7.09 & 0.48 \\
$\mathrm{In}$ & 430 & 15.74 & 0.68 \\
$\mathrm{La}$ & 1193 & 22.54 & 0.50 \\
$\mathrm{Mn}$ & 1517 & 7.35 & 0.50 \\
$\mathrm{Mo}$ & 2895 & 9.39 & 0.50 \\
$\mathrm{Nb}$ & 2740 & 10.81 & 0.50 \\
$\mathrm{Ni}$ & 1728 & 6.60 & 0.47 \\
$\mathrm{~Pb}$ & 601 & 18.28 & 0.55 \\
$\mathrm{Sb}$ & 904 & 16.96 & 0.40 \\
$\mathrm{Si}$ & 1687 & 8.61 & 0.38 \\
$\mathrm{Sn}$ & 505 & 16.30 & 0.64 \\
$\mathrm{Ta}$ & 3123 & 10.81 & 0.50 \\
$\mathrm{Ti}$ & 1998 & 10.58 & 0.50 \\
$\mathrm{~V}$ & 1973 & 8.36 & 0.50 \\
$\mathrm{~W}$ & 3655 & 9.55 & 0.50 \\
$\mathrm{Zn}$ & 693 & 9.17 & 0.54 \\
\hline
\end{tabular}

Table 2. Partial enthalpy of mixing $\Delta \bar{H}_{X(Y)} / \mathrm{kJ} \cdot \mathrm{mol}^{-1}$ calculated from Miedema's semi-empirical method [13].

\begin{tabular}{|c|c|c|c|c|c|c|c|c|}
\hline $\begin{array}{l}\text { Solvent } \\
(Y)\end{array}$ & $\begin{array}{l}\text { Solute } \\
(X)\end{array}$ & $\Delta \bar{H}_{X(Y)}$ & $\begin{array}{l}\text { Solvent } \\
(Y)\end{array}$ & $\begin{array}{c}\text { Solute } \\
(X)\end{array}$ & $\Delta \bar{H}_{X(Y)}$ & $\begin{array}{c}\text { Solvent } \\
(Y)\end{array}$ & $\begin{array}{l}\text { Solute } \\
(X)\end{array}$ & $\Delta \bar{H}_{X(Y)}$ \\
\hline $\mathrm{Bi}$ & $\mathrm{Cd}$ & 4 & $\mathrm{Bi}$ & In & -5 & $\mathrm{Bi}$ & $\mathrm{Pb}$ & 0 \\
\hline $\mathrm{Bi}$ & $\mathrm{Sb}$ & 3 & $\mathrm{Bi}$ & $\mathrm{Sn}$ & 5 & $\mathrm{Bi}$ & $\mathrm{Zn}$ & 14 \\
\hline $\mathrm{Cd}$ & $\mathrm{Bi}$ & 5 & $\mathrm{Cd}$ & $\mathrm{Pb}$ & 7 & $\mathrm{Cd}$ & $\mathrm{Sb}$ & -11 \\
\hline $\mathrm{Cd}$ & Sn & -1 & $\mathrm{Cd}$ & $\mathrm{Zn}$ & 3 & In & $\mathrm{Pb}$ & -3 \\
\hline In & Sn & -1 & In & $\mathrm{Zn}$ & 10 & $\mathrm{~Pb}$ & $\mathrm{Bi}$ & 0 \\
\hline $\mathrm{Pb}$ & $\mathrm{Cd}$ & 5 & $\mathrm{~Pb}$ & In & -3 & $\mathrm{~Pb}$ & $\mathrm{Sb}$ & 2 \\
\hline $\mathrm{Pb}$ & Sn & 6 & $\mathrm{~Pb}$ & $\mathrm{Zn}$ & 16 & $\mathrm{Sb}$ & $\mathrm{Cd}$ & -8 \\
\hline $\mathrm{Sb}$ & $\mathrm{Pb}$ & 2 & Sn & $\mathrm{Cd}$ & -1 & $\mathrm{Sn}$ & In & -1 \\
\hline Sn & $\mathrm{Pb}$ & 7 & $\mathrm{Sn}$ & $\mathrm{Zn}$ & 3 & $\mathrm{Zn}$ & $\mathrm{Bi}$ & 23 \\
\hline $\mathrm{Zn}$ & $\mathrm{Cd}$ & 4 & $\mathrm{Zn}$ & In & 14 & $\mathrm{Zn}$ & $\mathrm{Pb}$ & 25 \\
\hline $\mathrm{Zn}$ & $\mathrm{Sb}$ & -5 & $\mathrm{Zn}$ & Sn & 5 & $\mathrm{Fe}$ & Co & -2 \\
\hline $\mathrm{Fe}$ & $\mathrm{Cr}$ & -6 & $\mathrm{Fe}$ & $\mathrm{Cu}$ & 50 & $\mathrm{Fe}$ & $\mathrm{La}$ & 25 \\
\hline $\mathrm{Fe}$ & $\mathrm{Mn}$ & 1 & $\mathrm{Fe}$ & Mo & -9 & $\mathrm{Fe}$ & $\mathrm{Nb}$ & -70 \\
\hline $\mathrm{Fe}$ & $\mathrm{Ni}$ & -6 & $\mathrm{Fe}$ & $\mathrm{Pb}$ & 160 & $\mathrm{Fe}$ & $\mathrm{Si}$ & -75 \\
\hline $\mathrm{Fe}$ & Sn & 56 & $\mathrm{Fe}$ & $\mathrm{Ta}$ & -67 & $\mathrm{Fe}$ & $\mathrm{Ti}$ & -74 \\
\hline $\mathrm{Fe}$ & V & -29 & $\mathrm{Fe}$ & W & 0 & $\mathrm{Cr}$. & Co & -18 \\
\hline $\mathrm{Cr}$ & $\mathrm{Cu}$ & 49 & $\mathrm{Cr}$ & $\mathrm{Mn}$ & 8 & $\mathrm{Cr}$ & Mo & 2 \\
\hline $\mathrm{Cr}$ & $\mathrm{Ni}$ & -27 & $\mathrm{Cr}$ & $\mathrm{Pb}$ & 153 & $\mathrm{Cr}$ & $\mathrm{Si}$ & -87 \\
\hline $\mathrm{Cr}$ & Sn & 53 & $\mathrm{Cr}$ & V & -8 & $\mathrm{Mn}$ & $\mathrm{Co}$ & -21 \\
\hline $\mathrm{Mn}$ & $\mathrm{Cr}$ & 9 & $\mathrm{Mn}$ & $\mathrm{La}$ & 14 & $\mathrm{Mn}$ & Mo & 22 \\
\hline $\mathrm{Mn}$ & $\mathrm{Nb}$ & -17 & $\mathrm{Mn}$ & $\mathrm{Ni}$ & -33 & $\mathrm{Mn}$ & $\mathrm{Pb}$ & 38 \\
\hline $\mathrm{Mn}$ & $\mathrm{Si}$ & -120 & $\mathrm{Mn}$ & $\mathrm{Ta}$ & -17 & $\mathrm{Mn}$ & $\mathrm{Ti}$ & -36 \\
\hline $\mathrm{Mn}$ & V & -3 & $\mathrm{Mn}$ & W & 28 & & & \\
\hline
\end{tabular}

The calculated results of $\varepsilon_{\mathrm{B}}^{\mathrm{C}}, \eta_{\mathrm{B}}^{\mathrm{C}}$ and $\sigma_{\mathrm{B}}^{\mathrm{C}}$ in the above systems are shown in Table 3 together with the experimental values of $\varepsilon_{\mathrm{B}}^{\mathrm{C}}$ [14 to 17]. Figure 2 presents a comparison of the calculated $\varepsilon_{\mathrm{B}}^{\mathrm{B}}$ with their experimental values. As shown in Table 3 and Fig. 2 , most of the calculated $\varepsilon_{\mathrm{B}}^{\mathrm{C}}$ values have the same sign as those for the experimental values. Since the simple assumptions were
Table 3. Calculated results for $\eta_{\mathrm{B}}^{\mathrm{C}} \sigma_{\mathrm{B}}^{\mathrm{C}}$ and $\varepsilon_{\mathrm{B}}^{\mathrm{C}}$ in liquid ternary alloys with the experimental values of $\varepsilon_{\mathrm{B}}^{\mathrm{C}}$.

\begin{tabular}{|c|c|c|c|c|c|c|c|c|c|}
\hline No. & & $\begin{array}{c}\text { emer } \\
B\end{array}$ & & $\begin{array}{c}\text { Temp./ } \\
\text { K }\end{array}$ & $\begin{array}{l}/ \eta_{\mathrm{B}}^{\mathrm{C}} / \\
\mathrm{kJ} \cdot \mathrm{mol}^{-1}\end{array}$ & $\begin{array}{c}\sigma_{\mathrm{B}}^{\mathrm{C}} / \\
\mathrm{I} \cdot \mathrm{K}^{-1} \mathrm{~mol}^{-1}\end{array}$ & $\begin{array}{l}\varepsilon_{\mathrm{B}}^{\mathrm{C}} / \\
\text { (calc.) }\end{array}$ & $\begin{array}{l}\varepsilon_{\mathrm{B}}^{\mathrm{C}} / \quad \mathrm{f} \\
(\exp .)\end{array}$ & \\
\hline 1 & $\mathrm{Bi}$ & $\mathrm{Cd}$ & $\mathrm{Pb}$ & 773 & 3 & 0.7 & 0.4 & 1.62 & \\
\hline 2 & $\mathrm{Bi}$ & $\mathrm{Zn}$ & $\mathrm{Cd}$ & 723 & -14 & -3.3 & -1.9 & -0.3 & \\
\hline 3 & $\mathrm{Bi}$ & $\mathrm{Zn}$ & In & 723 & 5 & 1.0 & 0.7 & 2.2 & \\
\hline 4 & $\mathrm{Bi}$ & $\mathrm{Zn}$ & $\mathrm{Pb}$ & 723 & 11 & 2.2 & 1.6 & 1.4 & \\
\hline 5 & $\mathrm{Bi}$ & $\mathrm{Zn}$ & $\mathrm{Sb}$ & 723 & -22 & -5.2 & -3.0 & -5.4 & \\
\hline 6 & $\mathrm{Bi}$ & $\mathrm{Zn}$ & $\mathrm{Sn}$ & 723 & -14 & -2.5 & -2.0 & -0.5 & \\
\hline 7 & $\mathrm{Cd}$ & $\mathrm{Bi}$ & $\mathrm{Pb}$ & 773 & -12 & -2.8 & -1.5 & -3.2 & \\
\hline 8 & $\mathrm{Cd}$ & $\mathrm{Pb}$ & $\mathrm{Sb}$ & 773 & 6 & 1.4 & 0.8 & 0.0 & \\
\hline 9 & $\mathrm{Cd}$ & $\mathrm{Pb}$ & $\mathrm{Sn}$ & 773 & 0 & -0.3 & 0.0 & 2.86 & \\
\hline 10 & $\mathrm{Cd}$ & $\mathrm{Sn}$ & $\mathrm{Pb}$ & 773 & 1 & -0.1 & 0.2 & 2.86 & \\
\hline 11 & $\mathrm{Cd}$ & $\mathrm{Zn}$ & $\mathrm{Pb}$ & 805 & 15 & 3.1 & 1.9 & 0.95 & \\
\hline 12 & $\mathrm{Cd}$ & $\mathrm{Zn}$ & Sn & 805 & 3 & 0.8 & 0.3 & -1.10 & \\
\hline 13 & In & $\mathrm{Zn}$ & $\mathrm{Pb}$ & 805 & 18 & 3.7 & 2.2 & 1.00 & \\
\hline 14 & In & $\mathrm{Zn}$ & Sn & 805 & -4 & -0.7 & -0.5 & -0.63 & \\
\hline 15 & $\mathrm{~Pb}$ & $\mathrm{Bi}$ & $\mathrm{Cd}$ & 773 & -1 & -0.1 & -0.1 & -0.86 & \\
\hline 16 & $\mathrm{~Pb}$ & $\mathrm{Cd}$ & Sn & 773 & -12 & -2.4 & -1.6 & 1.35 & \\
\hline 17 & $\mathrm{~Pb}$ & $\mathrm{Sb}$ & $\mathrm{Cd}$ & 773 & -15 & -3.4 & -1.9 & -1.60 & \\
\hline 18 & $\mathrm{~Pb}$ & $\mathrm{Zn}$ & $\mathrm{Bi}$ & 805 & 7 & 1.6 & 0.9 & -0.70 & \\
\hline 19 & $\mathrm{~Pb}$ & $\mathrm{Zn}$ & $\mathrm{Cd}$ & 805 & -17 & -3.9 & -2.1 & -2.05 & \\
\hline 20 & $\mathrm{~Pb}$ & $\mathrm{Zn}$ & In & 805 & 1 & -0.1 & 0.2 & -1.90 & \\
\hline 21 & $\mathrm{~Pb}$ & $\mathrm{Zn}$ & $\mathrm{Sn}$ & 805 & -17 & -3.3 & -2.1 & -4.20 & 1 \\
\hline 22 & $\mathrm{Sb}$ & $\mathrm{Cd}$ & $\mathrm{Pb}$ & 773 & 13 & 3 & 1.6 & 2.8 & \\
\hline 23 & $\mathrm{Sn}$ & $\mathrm{Cd}$ & $\mathrm{Pb}$ & 773 & 1 & 0.5 & 0.1 & 0.0 & \\
\hline 24 & $\mathrm{Sn}$ & $\mathrm{Cd}$ & $\mathrm{Zn}$ & 973 & 1 & -0.1 & 0.1 & -1.65 & 1 \\
\hline 25 & Sn & $\mathrm{Zn}$ & In & 805 & 12 & 2.2 & 1.5 & 0.41 & \\
\hline 26 & $\mathrm{Sn}$ & $\mathrm{Zn}$ & $\mathrm{Pb}$ & 805 & 15 & 3.1 & 1.9 & 1.22 & \\
\hline 27 & $\mathrm{Fe}$ & Mn & $\mathrm{Co}$ & 1843 & -20 & -1.8 & -1.1 & -0.9 & 1 \\
\hline 28 & $\mathrm{Fe}$ & $\mathrm{Mn}$ & $\mathrm{Cr}$ & 1843 & 14 & 0.8 & 0.8 & 0.9 & \\
\hline 29 & $\mathrm{Fe}$ & $\mathrm{Mn}$ & $\mathrm{La}$ & 1873 & -12 & -0.9 & -0.7 & 61.6 & \\
\hline 30 & $\mathrm{Fe}$ & $\mathrm{Mn}$ & $\mathrm{Mo}$ & 1843 & 30 & 1.4 & 1.8 & 1.1 & \\
\hline 31 & $\mathrm{Fe}$ & $\mathrm{Mn}$ & $\mathrm{Nb}$ & 1843 & 52 & 2.9 & 3.0 & 2.1 & \\
\hline 32 & $\mathrm{Fe}$ & $\mathrm{Mn}$ & $\mathrm{Ni}$ & 1843 & -28 & -2.6 & -1.5 & -1.8 & \\
\hline 33 & $\mathrm{Fe}$ & $\mathrm{Mn}$ & $\mathrm{Pb}$ & 1823 & -123 & -16.5 & -6.1 & -5.2 & 1 \\
\hline 34 & $\mathrm{Fe}$ & $\mathrm{Mn}$ & $\mathrm{Si}$ & 1873 & -46 & -5.3 & -2.3 & -3.3 & \\
\hline 35 & $\mathrm{Fe}$ & $\mathrm{Mn}$ & $\mathrm{Ta}$ & 1843 & 49 & 2.3 & 2.9 & 0.4 & \\
\hline 36 & $\mathrm{Fe}$ & $\mathrm{Mn}$ & $\mathrm{Ti}$ & 1873 & 37 & 2.5 & 2.1 & $-9.7 *$ & 1 \\
\hline 37 & $\mathrm{Fe}$ & $\mathrm{Mn}$ & $\mathrm{V}$ & 1843 & 25 & 1.8 & 1.4 & 1.3 & \\
\hline 38 & $\mathrm{Fe}$ & $\mathrm{Mn}$ & $W$ & 1843 & 27 & 0.7 & 1.7 & 3.1 & \\
\hline 39 & $\mathrm{Fe}$ & $\mathrm{Cr}$ & $\mathrm{Co}$ & 1903 & -10 & -0.8 & -0.5 & -4.7 & \\
\hline 40 & $\mathrm{Fe}$ & $\mathrm{Cr}$ & $\mathrm{Cu}$ & 1873 & 5 & -1.5 & 0.5 & 4.1 & \\
\hline 41 & $\mathrm{Fe}$ & $\mathrm{Cr}$ & $\mathrm{Mn}$ & 1843 & 13 & 0.7 & 0.8 & 0.9 & \\
\hline 42 & $\mathrm{Fe}$ & $\mathrm{Cr}$ & Mo & 1873 & 17 & 3.1 & 0.7 & 0.0 & \\
\hline 43 & $\mathrm{Fe}$ & $\mathrm{Cr}$ & $\mathrm{Ni}$ & 1873 & -15 & -1.5 & -0.8 & 0.0 & \\
\hline 44 & $\mathrm{Fe}$ & $\mathrm{Cr}$ & $\mathrm{Pb}$ & 1873 & -1 & -5.5 & 0.6 & 4.4 & \\
\hline 45 & $\mathrm{Fe}$ & $\mathrm{Cr}$ & $\mathrm{Si}$ & 1903 & -6 & -2.0 & -0.1 & 0.0 & \\
\hline 46 & $\mathrm{Fe}$ & $\mathrm{Cr}$ & $\mathrm{Sn}$ & 1823 & 3 & -3.2 & 0.6 & 3.3 & \\
\hline 47 & $\mathrm{Fe}$ & $\mathrm{Cr}$ & V & 1873 & 27 & 2.8 & 1.4 & 2.6 & \\
\hline
\end{tabular}

*As Ueno et al. [18] pointed out, $\varepsilon_{\mathrm{Mn}}^{\mathrm{Ti}}(\exp )=.3.9[21]$.

used to extend the model to ternary systems in this work, it is difficult to make a reliable comparison of the calculated and experimental $\varepsilon_{\mathrm{B}}^{\mathrm{C}}$ results. In addition, the accuracy of the calculated results for $\varepsilon_{\mathrm{B}}^{\mathrm{C}}$ in this model depends on that of $\Delta \bar{H}_{X(Y)}(X=\mathrm{A}$, $\mathrm{B}$ or $\mathrm{C} ; Y=\mathrm{A}$ or $\mathrm{B})$ calculated from Miedema's semi-empirical method. 


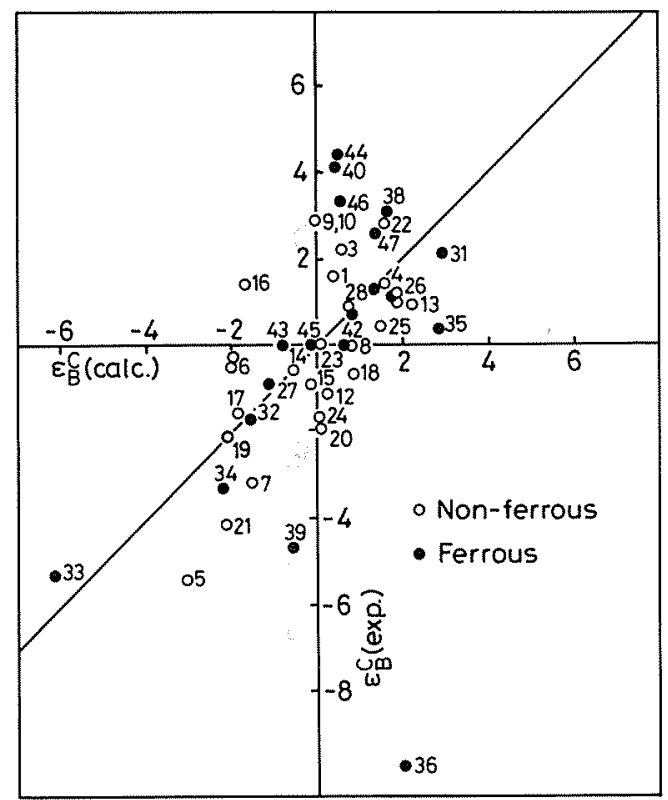

Fig. 2. Comparison of the calculated $\varepsilon_{\mathrm{B}}^{\mathrm{C}}$ with the experimental values (numbers in the figure correspond to those in Table 3).

Ueno et al. have also calculated $\varepsilon_{\mathrm{B}}^{\mathrm{C}}$ using Miedema's semiempirical method on the basis of the pseudopotential formalism coupled with the Gibbs energy of the hard sphere system [18, 19]. They did not, however, refer to the relationship between $\eta_{B}^{\mathrm{C}}$ and $\sigma_{\mathrm{B}}^{\mathrm{C}}$.

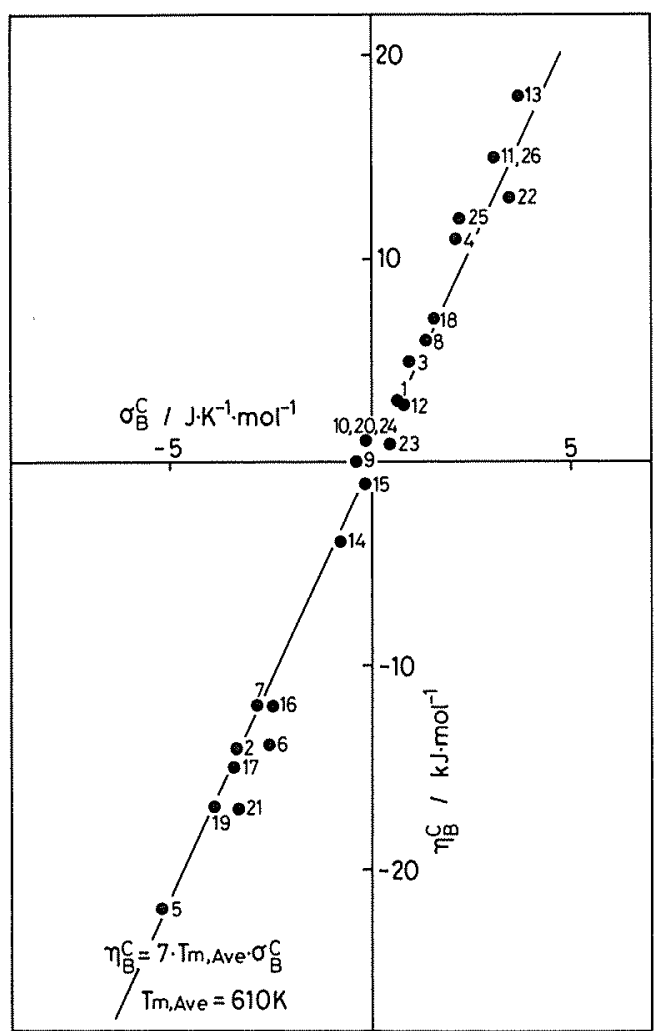

Fig. 3. Relationship between $\eta_{\mathrm{B}}^{\mathrm{C}}$ and $\sigma_{\mathrm{B}}^{\mathrm{C}}$ (calculated) for liquid non-ferrous ternary alloys (numbers in the figure correspond to those in Table 3).

\section{Relation Between Enthalpy and Entropy Interaction Parameters in Liquid Ternary Alloys}

Lupis and Elliott [9] and Richardson [20] have shown that certain correlations exist between $\eta_{\mathrm{B}}^{\mathrm{C}}$ and $\sigma_{\mathrm{B}}^{\mathrm{C}}$ in some liquid ternary systems on the basis of the available experimental values. Figures 3 and 4 show the relation between $\eta_{\mathrm{B}}^{\mathrm{C}}$ and $\sigma_{\mathrm{B}}^{\mathrm{C}}$ calculated from the present model in non-ferrous alloys and ferrous alloys, respectively. From these figures, there seem to be linear correlations between $\eta_{\mathrm{B}}^{\mathrm{C}}$ and $\sigma_{\mathrm{B}}^{\mathrm{C}}$. From Eqs. (25), (26), (32) and (33), if the components in the ternary alloys have similar physical properties, i.e.

$$
\begin{aligned}
\beta_{\mathrm{A}} & =\beta_{\mathrm{B}}=\beta_{\mathrm{C}}(=0.50) \\
V_{\mathrm{A}} & \approx V_{\mathrm{B}} \approx V_{\mathrm{C}} \\
T_{\mathrm{m}, \mathrm{A}} & \approx T_{\mathrm{m}, \mathrm{B}} \approx T_{\mathrm{m}, \mathrm{C}}\left(=T_{\mathrm{m}, \mathrm{Ave}}\right)
\end{aligned}
$$

the following approximate equation applies:

$\eta_{\mathrm{B}}^{\mathrm{C}} \approx 7.0 \cdot T_{\mathrm{m}, \text { Ave }} \cdot \sigma_{\mathrm{B}}^{\mathrm{C}}$

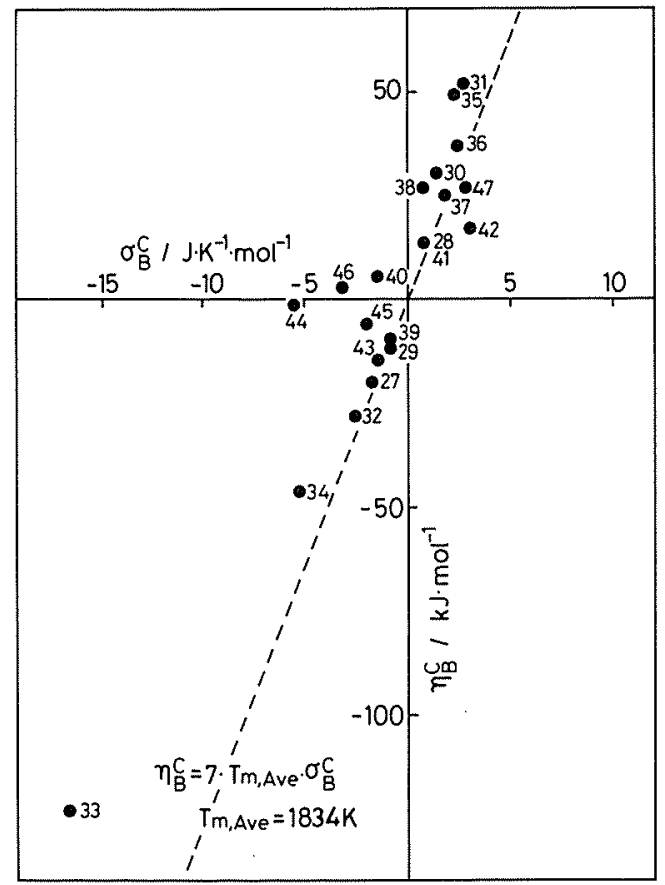

Fig. 4. Relationship between $\eta_{\mathrm{B}}^{\mathrm{C}}$ and $\sigma_{\mathrm{B}}^{\mathrm{C}}$ (calculated) for liquid ferrous ternary alloys (numbers in the figure correspond to those in Table 3 ).

where $T_{\mathrm{m}}$, Ave is the arithmetic average of $T_{\mathrm{m}, \mathrm{A}}, T_{\mathrm{m}, \mathrm{B}}$ and $T_{\mathrm{m}, \mathrm{C}}$.

The above equation implies that the alloy, for which the components have the higher melting points, will show a larger value of the ratio $\eta_{\mathrm{B}}^{\mathrm{C}} / \sigma_{\mathrm{B}}^{\mathrm{C}}$. The relation in Eq. (35) with the average value of the melting points of $\mathrm{Bi}, \mathrm{Cd}, \mathrm{In}, \mathrm{Pb}, \mathrm{Sb}, \mathrm{Sn}$ and $\mathrm{Zn}$ $\left(T_{\mathrm{m} \text { Ave }}=610 \mathrm{~K}\right)$ is shown in Fig. 3 , where the approximation in Eq. (35) satisfies the relation between $\eta_{\mathrm{B}}^{\mathrm{C}}$ and $\sigma_{\mathrm{B}}^{\mathrm{C}}$ for the alloys formed by the above metals. The dotted line in Fig. 4 indicates the relation in Eq. (35) with the average value of the melting point of $\mathrm{Fe}, \mathrm{Mn}$ and $\mathrm{Cr}\left(T_{\mathrm{m}, \mathrm{Ave}}=1834 \mathrm{~K}\right)$. As shown in this figure, when the melting point of the third element $\mathrm{C}$ is much different from those of $\mathrm{Fe}, \mathrm{Mn}$ and $\mathrm{Cr}$, their points (e.g., No. 33 for $\mathrm{Fe}-\mathrm{Mn}-\mathrm{Pb}$, No. 44 for $\mathrm{Fe}-\mathrm{Cr}-\mathrm{Pb}$ and so on) are located away from the line. Hence, if the second or third element 
in $\varepsilon_{\mathrm{B}}^{\mathrm{C}}$ is a gaseous element such as oxygen or nitrogen, another relationship between $\eta_{\mathrm{B}}^{\mathrm{C}}$ and $\sigma_{\mathrm{B}}^{\mathrm{C}}$ instead of Eq. (35) should be derived because $U_{X X}$ can not be expressed in terms of the melting points of those elements as in Eq. (33).

\section{Concluding Remarks}

In the present work, a previously described solution model based on the free volume theory was applied to ternary alloys to calculate the interaction parameter $\varepsilon_{\mathrm{B}}^{\mathrm{C}}$, as well as the enthalpy and entropy interaction parameters, $\eta_{\mathrm{B}}^{\mathrm{C}}$ and $\sigma_{\mathrm{B}}^{\mathrm{C}}$, in some non-ferrous and ferrous alloys. The values of $\varepsilon_{\mathrm{B}}^{\mathrm{C}}, \eta_{\mathrm{B}}^{\mathrm{C}}$ and $\sigma_{\mathrm{B}}^{\mathrm{C}}$ can be evaluated from the partial enthalpy of mixing $\Delta \tilde{H}_{X(Y)}$ obtained by Miedema's semi-empirical method and some physical properties of pure components. The relationship between $\eta_{\mathrm{B}}^{\mathrm{C}}$ and $\sigma_{\mathrm{B}}^{\mathrm{C}}$ can be expressed approximately as a linear relation dependent upon the melting points of the components.

\section{Literature}

1. Tanaka,T.; Gokcen,N.A.; Morita,Z.:Z. Metallkd. 81 (1990) 49.

2. Tanaka, T.; Gokcen, N.A.; Morita,Z.:Z. Metallkd. 81 (1990) 349.

3. Tanaka, T.; Gokcen, N. A.; Morita, Z.; Spencer, P.J.: Anales de Fisica-Serie B (Proceedings of the Discussion Meeting on Thermodynamics of Alloys, Barcelona, Spain) Vol. 86 (1990) 104.

4. Tanaka, T.; Gokcen, N. A.; Neuschïtz, D.; Spencer, P. J.; Morita, Z.: Steel Research 62 (1991) 385
5. Tanaka, T.; Imai, N.; Kiyose, A.; Iida, T.; Morita, Z.: Z. Metallkd. $82(1991) 836$

6. Shimoji, M.; Niwa, K.: Acta Metall. 5 (1957) 496.

7. Gokcen, N. A.: Statistical Thermodynamics of Alloys, Plenum Press, New York (1986)

8. Wagner, $C$.: Thermodynamics of Alloys, Addison-Wesley, Reading, MA (1952) 51

9. Lupis, C.H.P.; Elliott,J.F.: Acta Metall. 15(1967) 265.

10. Iida, T.; Guthrie, R.I.L.: The Physical Properties of Liquid Metals, Clarendon Press, Oxford (1988) 6, 17,97 and 100

11. Iida, T.; Kasama, A.; Misawa, M.; Morita, Z.: J. Japan Inst. Metals, 38 (1974) 177.

12. Kasama, A.; Iida, T.; Morita, Z.: J. Japan Inst. Metals 40 (1976) 1030.

13. Niessen, A. K.; de Boer, F. R.; Boom, R.; de Châtel, P. F.; Mattens, W. C.M.: Miedema,A.R.: CALPHAD 7 (1983) 51.

14. Dealy,J.M.; Pehlke, R.D.:.Trans. Met. Soc. AIME 227 (1963) 88.

15. Gluck, J. V.; Pehlke, R.D.: Trans. Met. Soc. AIME 239 (1967) 36.

16. Moser, Z.: Metall. Trans. B6B (1975) 103.

17. Steelmaking Data Sourcebook. The Japan Society for the Promotion of Science, The 19th Committee on Steelmaking, Gordon and Breach Science Pub., New York (1988) 273.

18. Ueno, S.; Waseda, Y.; Jacob, K. T.; Tamaki, S.: Steel Research 59 (1988) 474.

19. Ueno, S.; Waseda, Y.; Chang, Y. A.: Z. Metallkd. 79 (1988) 435.

20. Richardson, F.D.: Physical Chemistry of Melts in Metallurgy, Vol. I Academic Press. New York (1974) 190

21. Buzek, Z.: Hutn. Aktuality Recnik. 20 (1979) and in: Final Report on "Thermodynamic Data for Steelmaking" Commission of the European Communities, Luxembourg, No. 7820 (1982) 298.

(Received February 24, 1992) 